\title{
Energy efficient hierarchical based fish eye state routing protocol for flying Ad-hoc networks
}

\author{
Muhammad Abul Hassan, Syed Irfan Ullah, Abdus Salam, Arbab Wajid Ullah, Muhammad Imad, \\ Farhat Ullah \\ Department of Computing and Technology, Abasyn University Peshawar, Pakistan
}

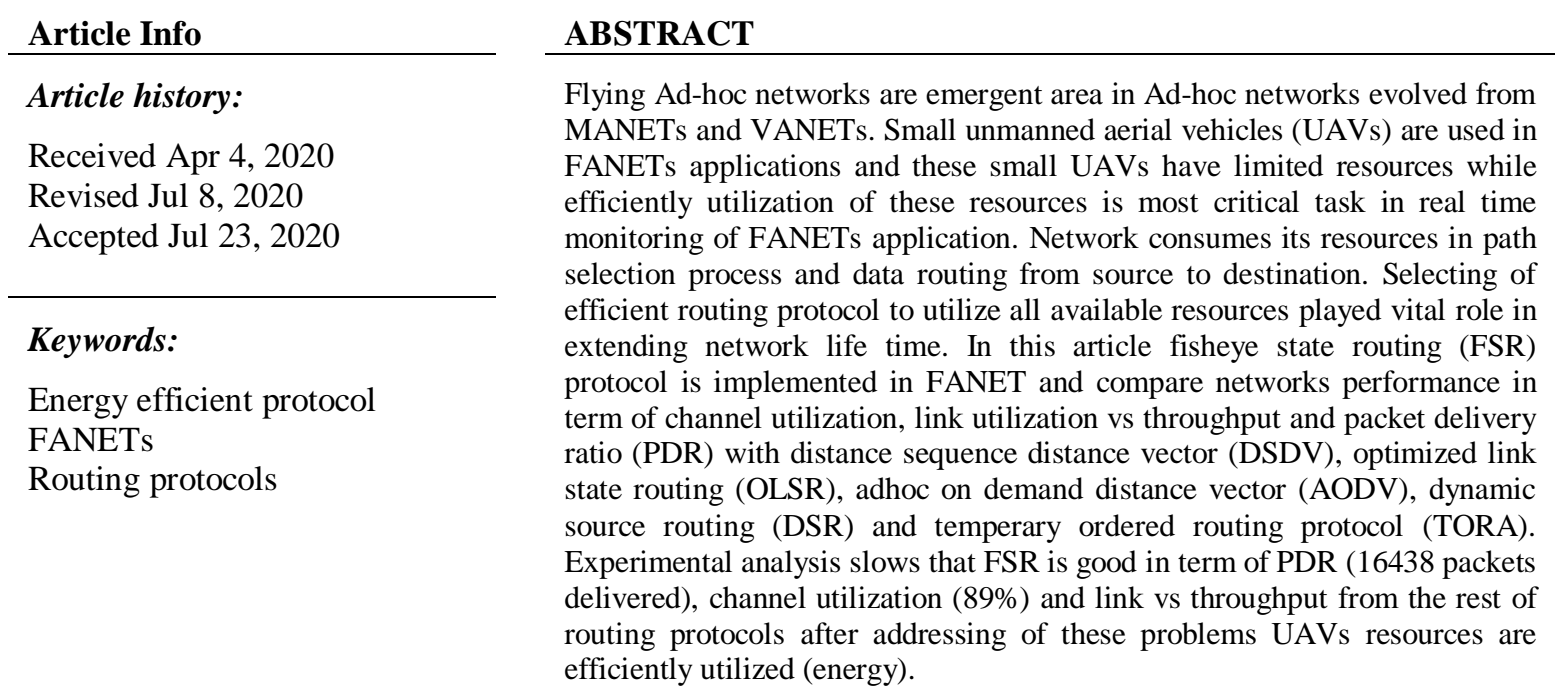

This is an open access article under the CC BY-SA license.

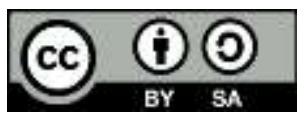

\section{Corresponding Author:}

Muhammad Abul Hassan

Department of Computer Science

Abasyn University Peshawar

Ring Road, Charsadda Link Near Patang Chowk Ashrafia Colony

Peshawar, Khyber Pakhtunkhwa 25000

Email: abulhassan900@gmail.com

\section{INTRODUCTION}

During last decades we have noticed large number of exponential growth in manufacturing industry of small unmanned aerial vehicles (UAVs) which resulting creation of another Ad-hoc network known as flying ad-hoc networks (FANETs). Due to exceptional features of FANETs (i) low cast (ii) easy deployment (iii) availability (iv) autonomy (v) versatility [1] it has gained more attention and is used to monitoring of boarder surveillance [2], disaster hit area monitoring [3], First ad services and also be used as relay nodes[4]. Apart from the monitoring FANETs also have the capabilities to collect data among UAVs network and send it to Base station [5]. Ad-hoc nature makes FANETs more power full even during the surveillance any UAV stop working apart from any reasonable reason still it has been connected to the network because of Ad-hoc nature [6]. FANETs also has the capabilities of self organizing and self-governing without any human intervention which make it better choice over other Ad-hoc networks [7]. Short range commination problem is encountered by multi point relay (MPR) node capabilities of flying Ad-hoc Networks [8]. FANETS can fly 30-460 (km/h) [9] with three dimensional (3D) area setting causing rapid topology changes which makes data routing between 
UAV and base station more difficult task while FANETs have limit resources e.g. Battery, Bandwidth [10]. FANETs is emerged from Mobile ad-hoc network and vehicular ad-hoc networks [11] so MANETs and VANETs data routing protocols were firstly implemented but movements of UAV does not adopted MANETs and VANETs routing protocols [12]. Three types of sub-networks are used to monitor geographical areas, Single UAV network in which there is one backbone UAV and rest of the UAV is connected to base station through this backbone UAV all data were aggregated and passed through this UAV backbone. Multiple UAV is another type which contains two backbone UAV each back bone is responsible to their respective cluster to collect data and send to the base station these clusters can communicate with each other via base station because they are not directly connected with each other and third type having more than two clusters and they can communicate with each because of the interconnected link between backbone UAVs [13]. Due to three dimensional mobility FANETs data routing protocol is sensitive to packet drop rate, Channel utilization and data routing protocol should be capable to conserve UAVs battery to enhance network life time[14]. In this research study fisheye state routing protocol (FSR) is implemented in FANETs to address routing problems. Fish eye routing protocol is an implicit hierarchical routing protocol [15]. It uses "fisheye" technique proposed by Klein Rock and Stevens in which scale of data is required to symbolize graphical information as shown in Figure 1. The eye of a fish captures greater pixels detail close to the focal point. In routing scheme, this method interprets to preserve accurate distance and course fine in sequence approximately the community of a node. It keeps the topology of a set of connections at each node however does not flood the entire community with information and exchanges topology information handiest with its pals [16, 17].

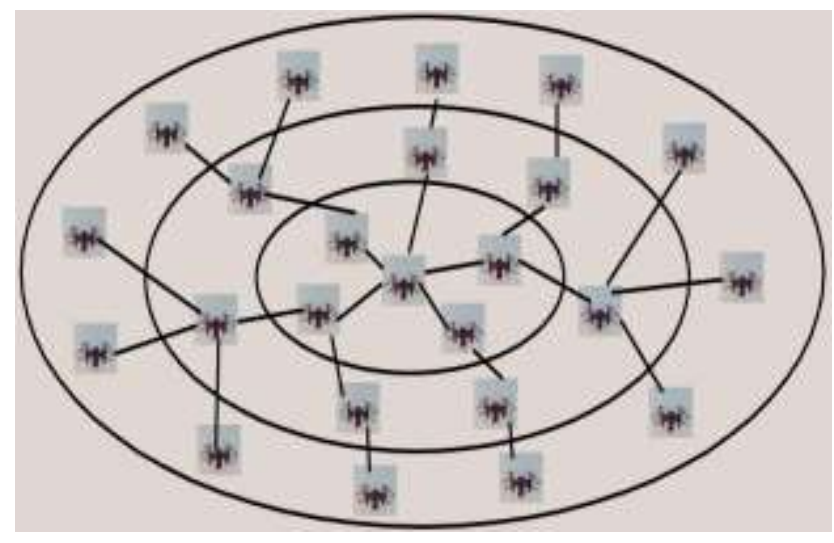

Figure 1. Fisheye state routing network hirarichy

\section{LITERATURE REVIEW}

We have studied reactive, proactive and hybrid routing protocols. Reactive routing protocols which are based on Demand strategies in which UAV want to convey data to another UAV in a network it established routing path for data delivery. Proactive Routing protocols are another protocol family In which all UAVs holds recent position of UAVs in a network. Hybrid routing protocols is a combination of both reactive and proactive strategies.

\subsection{Optimized link state routing protocol (OLSR)}

Proactive nature routing protocol specially designed for Ad-hoc networks in which each UAV holds routing table representing whole topology of UAV network. Every UAV shares their recent (up-to-date) geographical location to whole network through flooding. Point-to-Point communication problems is handled by introduction of Multi point relay UAVs distant UAV can communicate with each via Multi point relay. Problem faced by the OLSR is no. of Multi point relay itself and does not work properly in more dense UAV network causing delay problem and followed by overhead problems [18, 19].

\subsection{Distance sequence distance vector (DSDV)}

Table Driven Proactive nature routing protocol family created for Ad-hoc networks developed [20] where Each UAV holds topology table of whole network while Looing problem is removed by the updated sequence number strategy of UAVs. Even and odd method is used to recognize present location of the UAVs. Drawback of DSDV is more delay and congestion problem in a network which is not affordable in real time scenario like FANETs. 


\subsection{Adhoc on demand distance vector (AODV)}

Reactive routing protocol specially desinged for MANETs and applied in to FANETs [21]. When UAV want to communicate with other UAVs in a network it sends RREQ to the adjacent UAV and further it spreads in to network for detecting of destination UAV. Once destination UAV is find out it then reply back with a RREP. RERR is also used for the broken or missing links between sender and destination. Once UAV moves to another geographical location is broadcast its updated sequence no information of UAV is stored at adjacent UAV topology table [22].

\subsection{Dynamic source routing (DSR)}

Reactive routing protocol created for ad-hoc networks. This allows network to be self-organized and self-configured without any central infrastructure. Due to the reactive nature it extracts topology changes from the source route (datagram header). When a node want to communicate (route discovery) it flooded the request in a network and establish a connection between source to destination. Route maintenance is done by source node by continuous sensing when a node sense topology changes it broadcast route error packet, node which receive error packet cache it and detect route information [23].

\subsection{Temporally Ordered Routing Algorithm (TORA)}

This type of protocol is designed for wireless Ad-hoc network and based on hybrid approach both reactive and proactive features were adopted. It focused on Localization of control packets to avoid flooding which cause overhead problem $\mathrm{s}$ in a network and consume for network resources. TORA also have three type of working approach (i) first when nodes wants to communicate it establish suitable routing path avoiding loops (ii) second it also maintain its neighbor topology table by exchanging of special data packet with its neighbor (iii) Third, when nodes changes its respective position it shares it current position with respective Ip address [24, 25].

Table 1. Strength and weakness of different routing protocols

\begin{tabular}{|c|c|c|}
\hline $\begin{array}{l}\text { Routing } \\
\text { Protocols }\end{array}$ & Strength & Weakness \\
\hline DSDV & Loops in a network were removed & Network Facing delays and congestion problems \\
\hline OLSR & MPR addressed overhead problems in a Network. & Overhead and Delays in Dense Network \\
\hline AODV & Packet Delivery Ratio (PDR) is high & $\begin{array}{l}\text { Because of Discovery process of UAVs in a network, generate more } \\
\text { data delays in a network }\end{array}$ \\
\hline DSR & $\begin{array}{l}\text { More reliable path selecting and establishment from } \\
\text { source to destination. }\end{array}$ & Network facing Overhead problem \\
\hline TORA & Link faliour recongnition is added to network & $\begin{array}{l}\text { Because of hybrid nature discovery process of intra and inter routing } \\
\text { makes more congestion problem in a network }\end{array}$ \\
\hline
\end{tabular}

\section{METHODOLOGY}

Constant bit rate (CBR) application is used to generate constant bit rate traffic in network simulator 2 version 2.35 . Size of the data packet is set to 512 bytes. Flat grid topology model is used having $1000 \mathrm{x}$ 1000 x $1000 \mathrm{~m}$ size area. Two ray ground reflection model is used for Radio Wave propagation and our topology consists of 30 nodes and one base station with a fixed position having coordinates $(500,500)$ with 30 nodes. Random way point is used for the UAV movements having (Minimum Speed: 1m/s, Maximum Speed: $10 \mathrm{~m} / \mathrm{s}$, Pause Time: 1s). Topology of our simulation as shown in Figure 2.

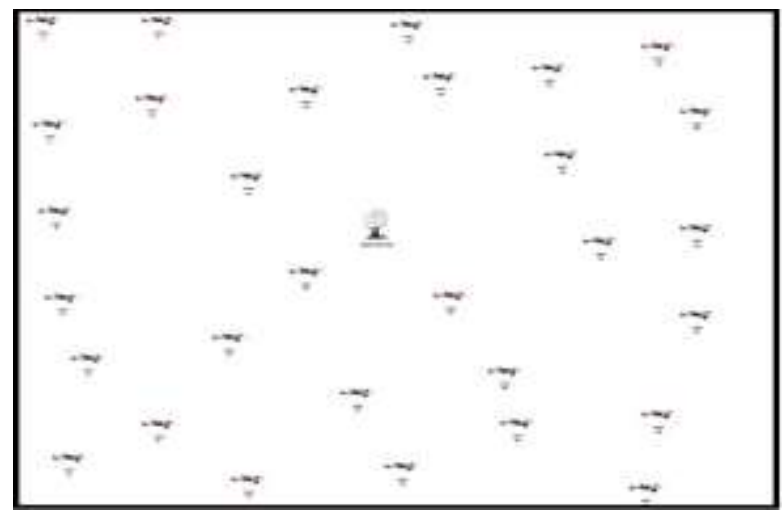

Figure 2. Topology of our simulation 


\section{RESULT AND ANALYSIS}

We have studied following performance parameters of a flying ad-hoc network.

a) Network/Channel Utilization (Bandwidth

b) Packet Delivery Ratio

c) Link Utilization vs Throughput

\subsection{Network/channel utilization (Bandwidth)}

Channel or link utilization means how much bandwidth of the channel/or link is being used. The overall bandwidth (date rate) or network utilization over a time is called network utilization or bandwidth. Network utilization in terms of bandwidth is measured as the consumption of network channel by all the nodes which can be calculated by given (1).

$$
\text { Network Utilization }=\frac{\Sigma \text { Size of sent packets }}{\text { time }}(\mathrm{Kbps})
$$

From the above Figure 3 we analyzed minimum, maximum and average channel utilization of routing protocols. TORA shows good result in channel utilization because of its hybrid nature while FSR and other protocols have relatively same results.

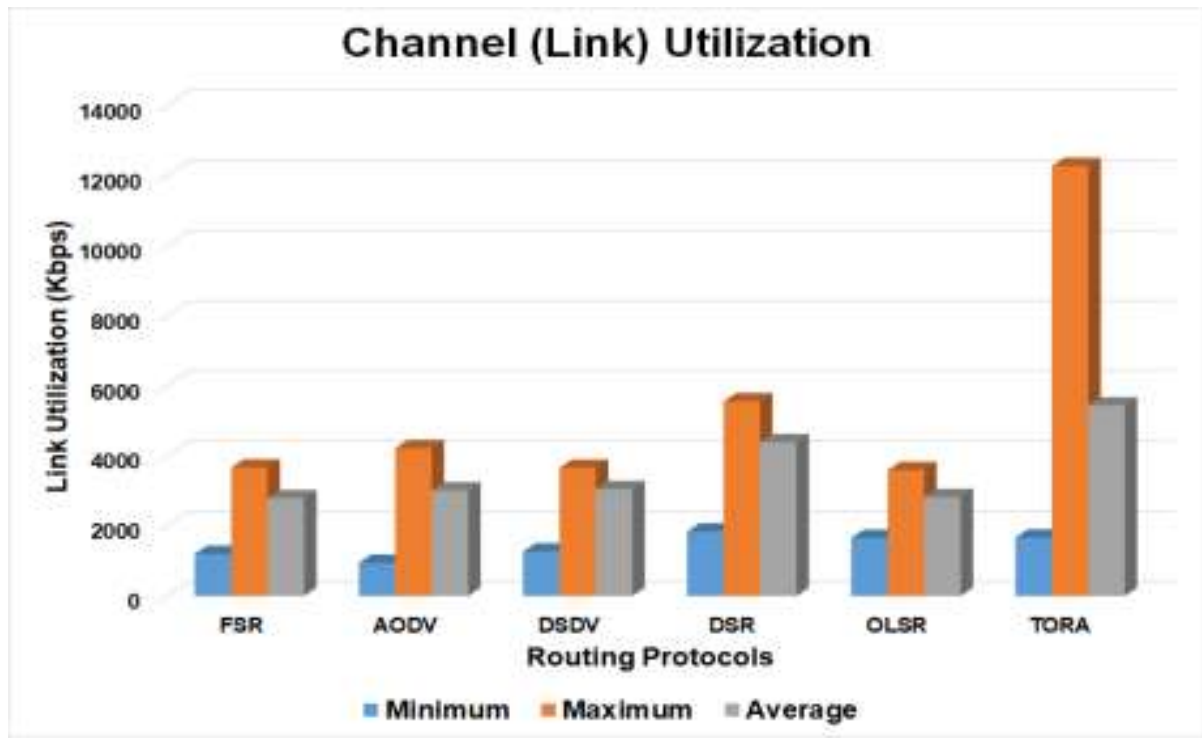

Figure 3. Channel or link utilization of routing protocols

\subsection{Link utilization vs throughput}

Link or channel utilization alone does not provide us accurate information which can be used later for design and development of FANET network. Therefore, Figure 4 comes into picture, in which we compared link/network utilization against actual network throughput. Figure 4 shows that TORA has high overhead as compared to all other routing protocols. From Figure 4 we analyzed that link utilization of FSR is $89 \%$ and throughput is (373.59) which is better then other routing protocols.

\subsection{Packet delivery ratio}

Packet delivered ratio is actually percentage of delivered packets. Packet delivery ratio can be calculated according to (2). Table 2 shows that Fisheye State Routing Protocol (FSR) has more Packet Delivery Ratio (PDR) then TORA, OLSR, DSR, AODV.

$$
\text { Packet Delivery Ratio }=\frac{\text { No.of packets received }}{\text { Total sent packets }}
$$




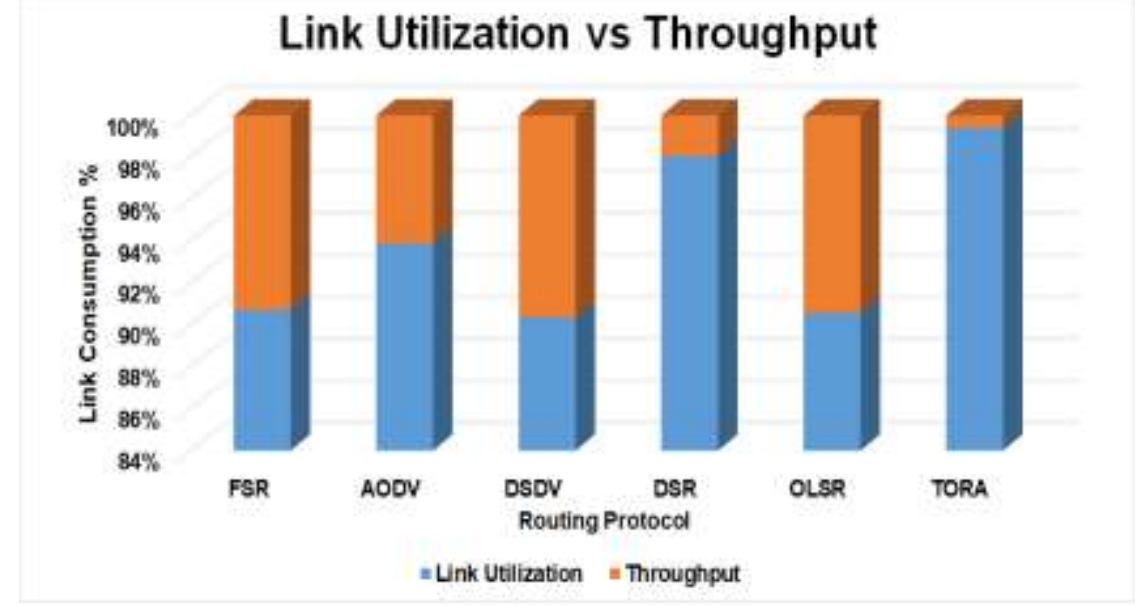

Figure 4. Link unitilization vs throughput of routing protocols

Table 2. PDR of all six routing protocols used in our study

\begin{tabular}{cccc}
\hline Protocols & Packets Sent & Packets Delivered & PDR $(\%)$ \\
\hline FSR & 51,069 & 16,438 & 32.19 \\
AODV & 51,069 & 12,014 & 23.53 \\
DSDV & 51,069 & 17,121 & 33.53 \\
DSR & 51,069 & 4,757 & 9.31 \\
OLSR & 51,069 & 16,371 & 32.06 \\
TORA & 51,069 & 3,096 & 6.06 \\
\hline
\end{tabular}

\section{CONCLUSION}

Flying Ad-hoc networks have limited battery and limited resources therefore we have to keep in mind while selecting routing protocol. Simulation results shows that fish Eye State Routing protocol has edge over other routing protocols in channel untilization (89\%), packet delivery Ratio (32\%) and link vs throughput and after improvements of these parametters UAV resource is efficiently utilized and network life time is enhanced.In future we will implement Fish Eye State Routing protocol in SANETs and RANETs with different mobility models with Enhanced Mobile Broadband (eMBB) and ultra-reliable and low latency commnication (URLLC).

\section{REFERENCES}

[1] I. Khan, I. Qureshi, M. Aziz, T. Cheema and S. Shah, "Smart IoT control-based nature inspired energy efficient routing protocol for flying ad hoc network (FANET)," IEEE Access, vol. 8, pp. 56371-56378, 2020.

[2] M. A. Khan, et al, "On the performance of flying ad-hoc networks (FANETs) with directional antennas," 20185 th International Multi-Topic ICT Conference (IMTIC), Jamshoro, 2018, pp. 1-8.

[3] A. Bujari, et al, "Flying ad-hoc network application scenarios and mobility models," International Journal of Distributed Sensor Networks, vol. 13, no. 10, pp. 1-16, 2017.

[4] O. Sahingoz, "Networking models in flying ad-hoc networks (FANETs): Concepts and Challenges," Journal of Intelligent \& Robotic Systems, vol. 74, no. 1-2, pp. 513-527, 2013.

[5] M. Khan, et al., "An efficient and provably secure certificateless key-encapsulated signcryption scheme for flying ad-hoc network," IEEE Access, vol. 8, pp. 36807-36828, 2020.

[6] M. Khan, et al, "An efficient and provably secure certificateless blind signature scheme for flying ad-hoc network based on multi-access edge computing," Electronics, vol. 9, no. 1, p. 30, 2019.

[7] M. A. Khan, A. Safi, I. Qureshi and I. Khan, "Flying ad-hoc networks (FANETs): A review of communication architectures and routing protocols," 2017 First International Conference on Latest trends in Electrical Engineering and Computing Technologies (INTELLECT), Karachi, 2017, pp. 1-9.

[8] W. Zafar and B. Khan, "A reliable, delay bounded and less complex communication protocol for multicluster FANETs," Digital Communications and Networks, vol. 3, no. 1, pp. 30-38, 2017.

[9] M. Khan, I. Khan, A. Safi and I. Quershi, "Dynamic routing in flying ad-hoc networks using topology-based routing protocols," Drones, vol. 2, no. 3, p. 27, 2018.

[10] A. Guillen-Perez and M. Cano, "Flying ad hoc networks: a new domain for network communications," Sensors, vol. 18, no. 10, p. 3571, 2018.

[11] K. Khullar, Y. Malhotra and A. Kumar, "Decentralized and Secure Communication Architecture for FANETs using Blockchain", Procedia Computer Science, vol. 173, pp. 158-170, 2020. Available: 10.1016/j.procs.2020.06.020.

\footnotetext{
Energy efficient hierarchical based fish eye state routing protocol for flying ... (Muhammad Abul Hassan)
} 
[12] O. Oubbati, M. Atiquzzaman, P. Lorenz, M. Tareque and M. Hossain, "Routing in Flying Ad Hoc Networks: Survey, Constraints, and Future Challenge Perspectives," IEEE Access, vol. 7, pp. 81057-81105, 2019.

[13] Y. Kwon and J. Hwang, "Mathematical modeling for flocking flight of autonomous multi-UAV system, including environmental factors," KSII Transactions on Internet and Information Systems, vol. 14, no. 2, pp. 595-609, 2020.

[14] M. Khan, et al, "Routing schemes in FANETs: A survey," Sensors, vol. 20, no. 1, p. 38, 2019.

[15] S. Kaur and S. Khurana, "Performance evaluation of FSR, DYMO and LANMAR routing protocols," International Journal of Computer Applications, vol. 82, no. 15, pp. 16-19, 2013.

[16] Pei, G., et al., "Fisheye state routing: a routing scheme for ad hoc wireless networks," 2000 IEEE International Conference on Communications. ICC 2000. Global Convergence Through Communications, vol. 1, pp.70-74, 2000.

[17] C. Yang and L. Tseng, "Fisheye zone routing protocol: A multi-level zone routing protocol for mobile ad hoc networks", Computer Communications, vol. 30, no. 2, pp. 261-268, 2007.

[18] Y. Jiao, W. Li and I. Joe, "OLSR improvement with link live time for FANETs," International Conference on Multimedia and Ubiquitous Engineering, Singapore, pp. 521-527, 2019.

[19] T. Clausen, P. Jacquet, "Optimized link state routing protocol (OLSR)," inria-00471712, 2003. [Online]. Available: https://hal.inria.fr/inria-00471712/

[20] A. G. Santiago, et al, "Evaluation of AODV and DSDV routing protocols for a FANET: Further results towards robotic vehicle networks," 9th Latin American Symposium on Circuits \& Systems (LASCAS), pp. 1-4, 2019.

[21] K. Darabkh, M. Alfawares and S. Althunibat, "MDRMA: Multi-data rate mobility-aware AODV-based protocol for flying ad-hoc networks," Science DIrect, 2019. [Online]. Available: https://www.sciencedirect.com/science/article/abs/pii/S2214209618303024.

[22] D. Cerri, A. Ghioni, "Securing AODV: The A-SAODV secure routing prototype," IEEE Communications Magazine, vol. 46, no. 2, pp. 120-125, 2008.

[23] H. Yang and Z. Liu, "An optimization routing protocol for FANETs," EURASIP Journal on Wireless Communications and Networking, vol. 2019, no. 1, pp. 1-8, 2019.

[24] V. Park and M. S. Corson, "Temporally-ordered routing algorithm (TORA) version 1 functional specification," Internet-Draft, 1997.

[25] D. Kaur and N. Kumar, "Comparative analysis of AODV, OLSR, TORA, DSR and DSDV routing protocols in mobile ad-hoc networks," International Journal of Computer Network and Information Security, vol. 5, no. 3, pp. 39-46, 2012.

\section{BIOGRAPHIES OF AUTHORS}

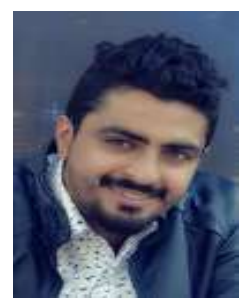

Muhammad Abul Hassan has Recieved BCS degree from Abdul Wali Khan University Mardan now pursuing Master in Computer Science (MSCS) from Abasyn University Peshawar Pakistan. He has Published Several International Research articles. His research interests include Network System Security, Intrusion Detection, Intrusion Prevention, Cryptography, Optimization Techniques, WSN, the IoT, and Mobile Ad-hoc Networks (MANETS), Flying Ad-hoc Networks (FANETS) and Artificial Intelligence.

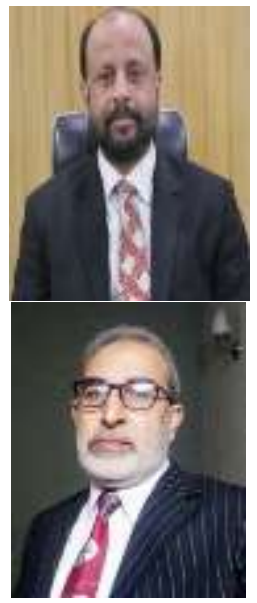

Dr. Syed Irfan Ullah received his master degree from University of Peshawar and received his Mphil and PhD from Islamic International University Islamabad. His field of research is Data and Network Security, Secure algorithm Design, Securing data on Private and public channels, Secure Communication, Cryptanalysis and Breaking code. Currently, he is doing his job as Assistant Professor in Abasyn University Peshawar and is supervising MS and PhD research Projects.

Dr. Abdus Salam has received his Master Degree from Quaid-e-Azam University Islamabad, Pakistan and Completed his Phd from International Islamic University Islamabad, Pakistan. He has published Several International Journals and Conferences. Abdus Salam is associate professor in the department of computing, Abasyn University Peshawar. His research focuses on a wide range of computing problems, from data acquisition and processing to information mining to software engineering processes and information security. He is particularly interested in making computers do more with less help from us, learn from experience, adapt effortlessly, and discover new knowledge. 


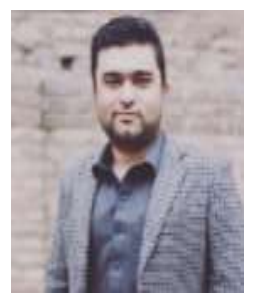

Arbab Wajid Ullah Khan currently working as Lecturer in Department of Computing and Technology, Abasyn University Peshawar campus, Pakistan. He received his BS degree in Information Technology from University of Peshawar and Master degree in Telecommunication and Networking from Abasyn University, Peshawar. Now pursuing his Phd In Computer Science from Comsats University Islamabad, Wah Compus Pakistan. He has published Several Journals and Conferences. His research Interests Include Mobile Ad-hoc Network (MANET), Wireless Body Area Networks (WBAN) and Software Defined Networks.

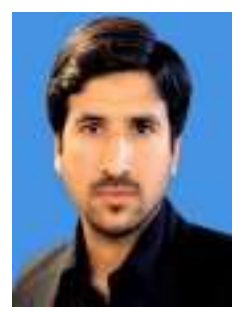

Muhammad Imad received his BS degree from University Of Engineering And Technology, Peshawar and now pursuing Master degree (MS-CS) from Abasyn University Peshawar, Pakistan. He has published several research articles in different international journlas. His area of interest in Image Processing, Machine Learning, Artificial Intelligence and SDN.

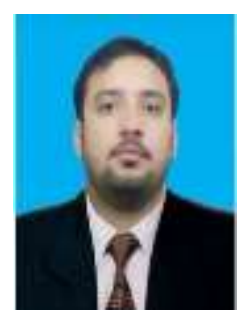

Farhat has recived BS degree form univeristy of malakand in Bs.IT now pursuing Master degree (MS-CS) form Abasyn University Peshwar Pakistan. He has published several Articles in International Journals. His Area of Interest in Processing, machine learning, deep learning and SDN. 